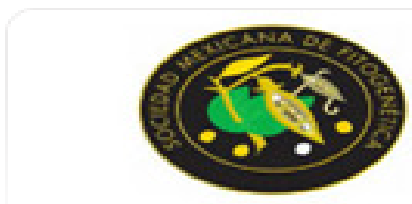

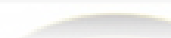

Revista Fitotecnia Mexicana

ISSN: 0187-7380

revfitotecniamex@gmail.com

Sociedad Mexicana de Fitogenética, A.C.

México

Vázquez-Carrillo, M. Gricelda; Arellano-Vázquez, José L.; Santiago-Ramos, David RENDIMIENTO Y CALIDAD DE GRANO Y TORTILLA DE MAÍCES HÍBRIDOS DE

VALLES ALTOS DE MÉXICO CRECIDOS EN RIEGO Y TEMPORAL

Revista Fitotecnia Mexicana, vol. 38, núm. 1, 2015, pp. 75-83

Sociedad Mexicana de Fitogenética, A.C.

Chapingo, México

Disponible en: http://www.redalyc.org/articulo.oa?id=61035375010

Cómo citar el artículo

- Número completo

- Más información del artículo

- Página de la revista en redalyc.org

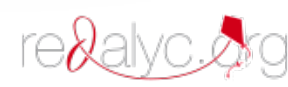

Sistema de Información Científica

Red de Revistas Científicas de América Latina, el Caribe, España y Portugal Proyecto académico sin fines de lucro, desarrollado bajo la iniciativa de acceso abierto 


\title{
RENDIMIENTO Y CALIDAD DE GRANO Y TORTILLA DE MAÍCES HÍBRIDOS DE VALLES ALTOS DE MÉXICO CRECIDOS EN RIEGO Y TEMPORAL
}

\author{
YIELD AND QUALITY OF GRAIN AND TORTILLA OF IRRIGATED AND RAINFED \\ HYBRID MAICES GROWN IN THE HIGH VALLEYS OF MÉXICO
}

\author{
M. Gricelda Vázquez-Carrillo ${ }^{1 \star}$, José L. Arellano-Vázquez¹ y David Santiago-Ramos ${ }^{2}$
}

\begin{abstract}
${ }^{1}$ Campo Experimental Valle de México, Instituto Nacional de Investigaciones Forestales, Agrícolas y Pecuarias (INIFAP). Km. 13.5 Carr. Los Reyes-Texcoco, Coatlinchán, 56250. Texcoco, Edo. de México. Tel. 01(595) 9212726. ${ }^{2}$ Programa de Posgrado en Alimentos del Centro de la República (PROPAC), Facultad de Química, Universidad Autónoma de Querétaro. Cerro de las Campanas S/N, Col. Las Campanas, 76010. Querétaro, Querétaro.
\end{abstract}

${ }^{*}$ Autor para correspondencia (gricelda_vazquez@yahoo.com)

\section{RESUMEN}

En México no existen estudios que permitan establecer los efectos del régimen hídrico en el cultivo sobre la calidad comercial del grano de maíz (Zea mays L.) y de la tortilla elaborada con granos de maíz de híbridos antiguos y modernos. Se estudió el efecto del régimen hídrico, tipo de madurez y genotipo en el rendimiento y calidad de grano y tortilla de híbridos de maíz de Valles Altos. Los híbridos cultivados en temporal presentaron menores rendimientos $(5.5 \mathrm{t}$ $\mathrm{ha}^{-1}$ ) que los cultivados en riego $\left(8.1 \mathrm{t} \mathrm{ha}^{-1}\right)$; además, los de temporal tuvieron granos más suaves, pequeños y con bajo peso hectolítrico $\left(<74 \mathrm{~kg} \mathrm{hL}^{-1}\right)$. En riego, los híbridos precoces y modernos (menos de 10 años de liberación) tuvieron mayor rendimiento $\left(9.1\right.$ y $8.7 \mathrm{t} \mathrm{ha}^{-1}$, respectivamente) y granos más duros (índice de flotación: 60 y $57 \%$ ) que los híbridos tardíos y antiguos. En la calidad de nixtamal y tortillas solo la variable porcentaje de sólidos en nejayote fue afectada $(\mathrm{P}<0.05)$ por los tres factores investigados. Los cambios en el pericarpio retenido en el nixtamal, así como la humedad, textura y rendimiento de las tortillas fueron poco afectados por los factores de estudio. Los híbridos modernos superaron a los antiguos en rendimiento y la precocidad, así como en el tamaño y la dureza del grano, pero la calidad de las tortillas se mantuvo igual en ambos grupos.

Palabras clave: Zea mays, calidad de grano, calidad de tortilla, rendimiento de grano, régimen hídrico.

\section{SUMMARY}

No studies in México have determined the effects of watering regime on commercial quality of maize grain and tortillas made from modern and old maize hybrids (Zea mays L.). The effect of watering regime on these genotypes was measured to determine how it affects yield and quality of grain and tortillas. The hybrids cultivated under rainfed conditions had lower yields $\left(5.5 \mathrm{t} \mathrm{ha}^{-1}\right)$ than those cultivated with irrigation $\left(8.1 \mathrm{t} \mathrm{ha}^{-1}\right)$. Moreover, under rainfed conditions hybrids had smaller and softer kernels with lower test weight $\left(<74 \mathrm{~kg} \mathrm{hL}^{-1}\right)$, than under irrigation where the modern hybrids (less than 10 years after their release) had higher yields (9.1 and $8.7 \mathrm{t} \mathrm{ha}^{-1}$ respectively). Additionally, their kernels were harder (flotation index: 60 and 57 $\%)$ than those from late and older hybrids. In nixtamal and tortilla quality, only the percent of solids in nejayote (residual liquid produced by nixtamal production) was affected $(P<0.05)$ by the three factors assessed. Changes in the pericarp retained in the nixtamal, as well as tortillas moisture, texture and yield were only slightly affected by these factors. Modern maize hybrids surpassed older ones in grain yield and earliness, as well as in kernel size and hardness, but tortilla quality was the same in both maize groups.

Index words: Zea mays, kernel quality, tortilla quality, grain yield, hydric regime.

\section{INTRODUCCIÓN}

En México se siembran anualmente alrededor de 8.4 millones de hectáreas de maíz (Zea mays L.), de las cuales $85.5 \%$ se cultivan bajo condiciones de temporal (secano) y el resto en condiciones de riego, con un rendimiento promedio de 2.2 y $5.7 \mathrm{t} \mathrm{ha}^{-1}$, respectivamente (SIAP, 2012). El bajo rendimiento promedio se debe principalmente a la deficiencia de agua durante la etapa reproductiva de la planta, entre la floración y el llenado de grano, lo que disminuye el rendimiento, número, peso y calidad del grano (Vázquez et al., 2012).

Al restringir el agua hasta el punto de marchitez de la planta en época de floración femenina el rendimiento de grano se reduce en $53 \%$, y cuando el marchitamiento ocurre tres semanas después de la floración la reducción es de 30 \% (Payero et al., 2006). Según NeSmith y Ritchie (1992), a los $7 \mathrm{~d}$ después de la floración femenina ocurre la mayor sensibilidad al déficit de agua, por lo que el número de granos por planta se puede reducir $45 \%$, mientras que entre los 12 y $16 \mathrm{~d}$ después de la floración la sequía reduce el peso de grano $51 \%$ respecto al testigo. Las condiciones de fertilidad del suelo también determinan el nivel del rendimiento en maíz (Kibet et al., 2009).

En híbridos de maíz de los Valles Altos de México, se observó que la fertilización nitrogenada adicionada a través del agua de riego incrementó las pérdidas de sólidos en el nejayote (solución de cocción), pero disminuyó el índice de flotación y el pericarpio retenido (Zepeda et al., 2007). Por ello en este estudio se evaluaron dos regímenes de humedad (temporal y riego), así como genotipos de diferentes 
generaciones y con diferente ciclo biológico.

Se sabe que la calidad comercial del grano de maíz está determinada por sus características físicas y su composición química. La industria de harinas nixtamalizadas (IHN) requiere maíces de endospermo duro o muy duro (índice de flotación $<40 \%$ ) y una humedad de nixtamal entre 36 y $42 \%$, mientras que la industria que elabora tortillas a partir de masa fresca (IMT) requiere maíces más suaves y con alto porcentaje de pericarpio remanente. Ambas industrias requieren granos con un peso hectolítrico mayor a 74 $\mathrm{kg} \mathrm{hL}^{-1} \mathrm{y}$ una pérdida de sólidos en nejayote menor de $5 \%$ (Vázquez et al., 2012). El objetivo fue estudiar el efecto del régimen hídrico del cultivo, tipo de madurez y genotipo, en el rendimiento y la calidad del grano y tortilla de híbridos de maíz de Valles Altos de México.

\section{MATERIALES Y MÉTODOS}

\section{Sitio experimental}

Los experimentos se establecieron en un campo experimental ubicado en Santa Lucía, Coatlinchán, Texcoco, Estado de México ( $19^{\circ} 29^{\prime} \mathrm{N}, 98^{\circ} 51^{\prime} \mathrm{O}$ ), a una altitud de 2240 $\mathrm{m}$. En el sitio de cultivo predominan los suelos cambrisoles de textura migajón arcillo-arenoso, con bajo contenido de materia orgánica y con pH ácido (Arellano et al., 1996).

\section{Material genético}

Se utilizaron 13 híbridos de maíz cuyas fuentes principales de germoplasma son la raza Chalqueño y en menor proporción germoplasma templado-subtropical; se clasificaron de acuerdo con su tipo de madurez en tardíos, intermedios y precoces. Los híbridos tardíos corresponden a híbridos generados hace 60 años y se les denominó "tardíos antiguos" y los generados hace 5 años "tardíos modernos". Los híbridos de madurez intermedia y los precoces se generaron hace menos de 10 años y se les denominó "intermedios-modernos" y "precoces-modernos", respectivamente (Cuadro 1).

\section{Diseño experimental}

Se utilizó el diseño de tratamientos de parcelas subdivididas con el diseño experimental de bloques completos al azar con tres repeticiones. La parcela grande estuvo constituida por el régimen hídrico del cultivo, la parcela media por el tipo de madurez y la parcela chica por los genotipos dentro de cada nivel de madurez.

\section{Manejo agronómico}

Los 13 híbridos se sembraron durante el ciclo primaveraverano de 2011, bajo el régimen hídrico de riego y temporal; en el caso de riego la siembra y el primer riego se hicieron el 18 de mayo, y los riegos subsecuentes se aplicaron el 25 de mayo, el 6 y el 14 de junio; la lámina de riego inicial fue de $20 \mathrm{~cm}$ y las restantes de $10 \mathrm{~cm}$. En el caso de temporal la siembra se realizó el 19 de junio y la lluvia registrada desde la siembra a la cosecha fue de $483 \mathrm{~mm}$. Durante el ciclo de cultivo se presentaron cuatro periodos de lluvias: el primer periodo ocurrió del 25 de junio hasta el 9 de agosto; el segundo periodo se dio del 26 de agosto al 5 de septiembre;

Cuadro 1. Identificación de los 13 híbridos de maíz, genealogía, tipo de madurez y antigüedad de obtención. Santa Lucia, Coatlinchán, Estado de México, ciclo Primavera-Verano 2011.

\begin{tabular}{lccc}
\hline Híbrido & Genealogía & Tipo de madurez & Antigüedad de obtención (años) \\
\hline H-1 & Chalqueño & Tardío-antiguo & 60 \\
H-2 & Chalqueño & Tardío-antiguo & 60 \\
H-5 & Chalqueño & Tardío-antiguo & 60 \\
H-64 & Chalqueño/Cónico x templado & Intermedio-moderno & 10 \\
H-68 & Chalqueño/Cónico x templado & Intermedio-moderno & 10 \\
H-70 & Chalqueño/Cónico x templado & Intermedio-moderno & 10 \\
H-72 & Chalqueño/Cónico x templado & Intermedio-moderno & 10 \\
HPrecoz-1 & Chalqueño/Cónico x templado & Precoz-moderno & 5 \\
HPrecoz-2 & Chalqueño/Cónico x templado & Precoz-moderno & 5 \\
HPrecoz-3 & Chalqueño/Cónico x templado & Precoz-moderno & 5 \\
HAzul-1 & Chalqueño x Bolita x Hibrido Valles Altos & Tardío-moderno & 5 \\
HGF-1002 & Chalqueño x Subtropical & Tardío-moderno & 5 \\
HV-65 & Chalqueño x Subtropical & Tardío-moderno & 5 \\
\hline
\end{tabular}


el tercero se presentó entre el 4 y 14 de octubre; y el último del 20 al 21 de octubre. Las condiciones de precipitación, temperaturas máximas y las etapas importantes durante el ciclo de cultivo en ambos regímenes hídricos se muestran en las Figuras 1 y 2. La fertilización se hizo con la fórmula $120 \mathrm{~N}-60 \mathrm{P}-30 \mathrm{~K}$ que indica las cantidades aplicadas de nitrógeno $(\mathrm{N})$, fósforo $\left(\mathrm{P}_{2} \mathrm{O}_{2}\right)$ y potasio $\left(\mathrm{K}_{2} \mathrm{O}\right)$ en $\mathrm{kg} \mathrm{ha}^{-1}$; en la siembra se aplicó la mitad del nitrógeno, todo el fósforo y potasio, y a los $40 \mathrm{~d}$ después se aplicó el nitrógeno restante. La cosecha se realizó los días 24 y 26 de noviembre para los híbridos de riego y temporal, respectivamente.

La humedad del grano a la cosecha fue de aproximadamente $24 \pm 2 \%$. Las mazorcas se colocaron en arpillas (costales de plástico), las cuales se colocaron verticalmente en un patio para permitir el secado de las mazorcas por exposición al aire y calor del sol; bajo estas condiciones el grano alcanzó una humedad entre 12 y $14 \%$ en dos meses, posteriormente se desgranó y se almacenó.

Todos los datos de precipitación y temperatura máxima y mínima se obtuvieron de una estación meteorológica cercana, ubicada en el Colegio de Postgraduados, Campus Montecillo.

\section{Rendimiento y características físicas y químicas de grano}

El rendimiento se evaluó como lo describieron Vázquez et al. (2012) y se expresó en tha-1 ${ }^{-1}$. En el laboratorio todas las muestras fueron ajustadas a $12 \%$ de humedad para realizar la caracterización físico-química. Se evaluó el peso de 100 granos (PCG) en una balanza analítica Sartorius ${ }^{\circledR}$ BL610 (Goettingen, Alemania), peso hectolítrico (PH) (método 55-10.01; AACC, 2000) e índice de flotación (IF) como medida indirecta de la dureza del grano (SE, 2002). En grano entero se determinó el contenido de proteína (método 4611.02; AACC, 2000), de almidón (Galicia et al., 2012) y de amilosa (Hoover y Ratnayake, 2001).

\section{Características de calidad de nixtamal y tortillas}

La nixtamalización y elaboración de las tortillas se hizo mediante el método descrito por Vázquez-Carrillo et al. (2011). El porcentaje de sólidos (PS) en nejayote se calculó con la materia seca residual después de haber evaporado el nejayote y el agua de lavado, y el pericarpio retenido (PR) se determinó mediante método gravimétrico (Vázquez-Carrillo et al., 2011). En nixtamal y tortillas se evaluó la humedad

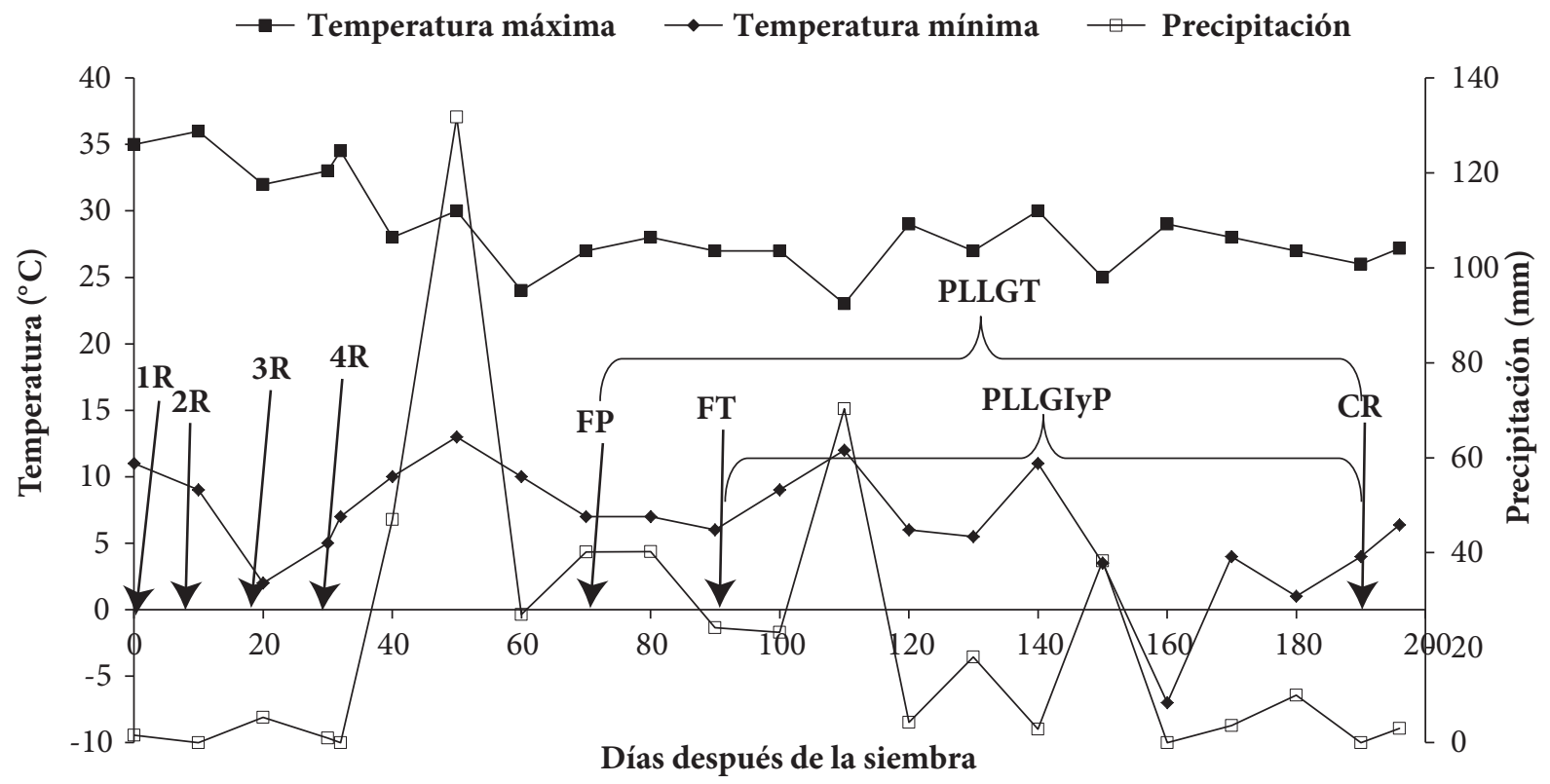

Figura 1. Temperaturas máximas y mínimas, precipitación y principales eventos climáticos durante el ciclo de cultivo en condiciones de riego. Santa Lucía, Estado de México, ciclo Primavera-Verano 2011. 1R = primer riego; $2 \mathrm{R}=$ segundo riego; $3 \mathrm{R}=$ tercer riego; $4 \mathrm{R}$ = cuarto riego; $\mathrm{FP}$ = floración de híbridos precoces; $\mathrm{FT}$ = floración híbridos tardíos; $\mathrm{PLLGIyP}=$ periodo de llenado de grano de híbridos intermedios y precoces; PLLGT = periodo de llenado de grano de híbridos tardíos; CR = cosecha. 


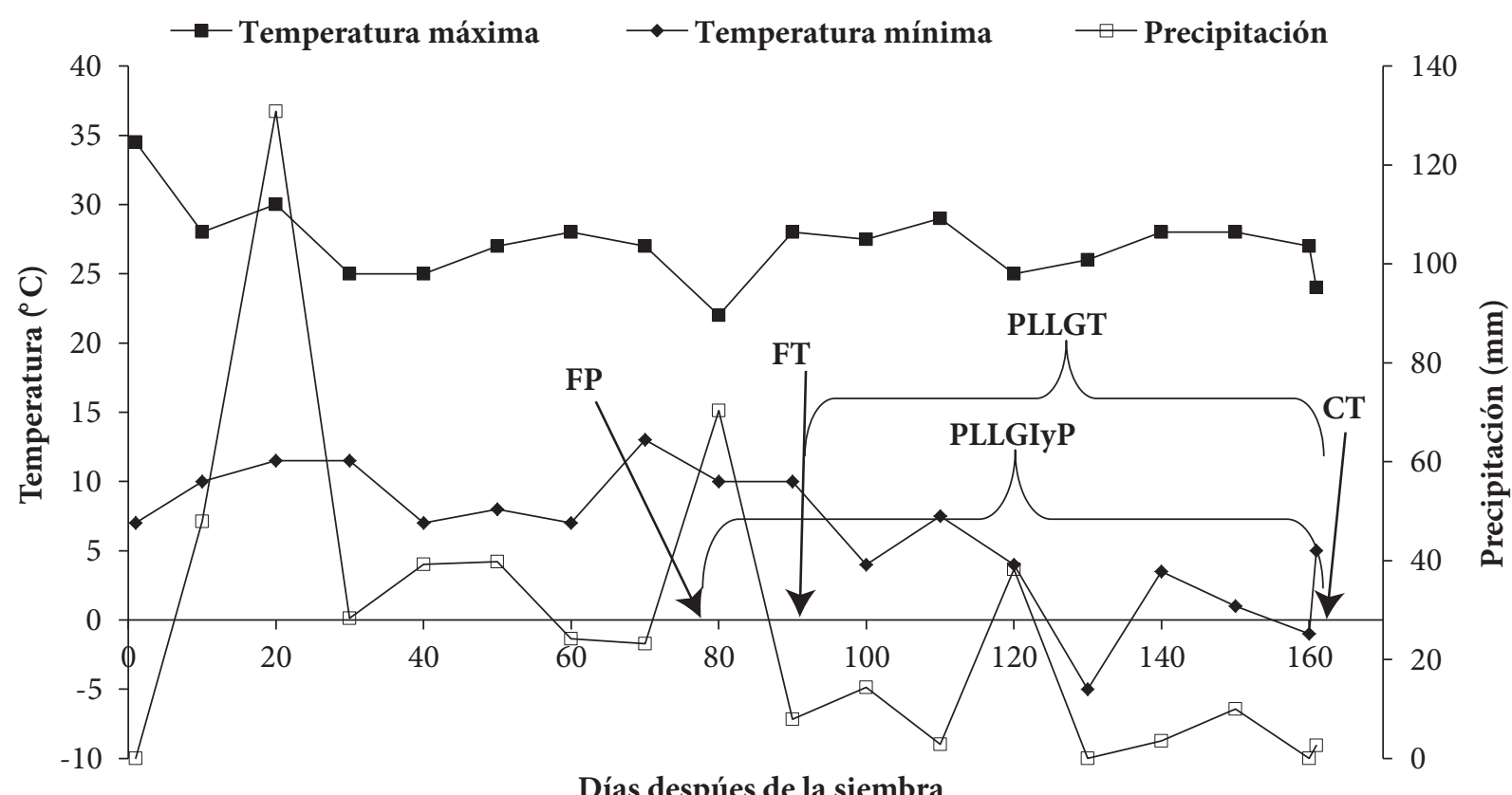

Figura 2. Temperaturas máximas y mínimas, precipitación y principales eventos climáticos durante el ciclo de cultivo en condiciones de temporal. Santa Lucía, Estado de México, ciclo Primavera-Verano 2011. FP = floración de híbridos precoces; FT = floración híbridos tardíos; PLLGIyP = periodo de llenado de grano de híbridos intermedios y precoces; PLLGT = periodo de llenado de grano de híbridos tardíos; $\mathrm{CT}$ = cosecha.

por el método 44-15.02 (AACC, 2000). El rendimiento de tortilla fría se cuantificó con base en la cantidad de tortilla obtenida por kilogramo de maíz procesado (Vázquez et al., 2012). En tortillas frías ( $2 \mathrm{~h}$ después de elaboradas) se evaluó la fuerza requerida para romperla, con el texturómetro Brookfield ${ }^{\circledR}$ (modelo CT3; Middleboro, MA, USA). Se trabajó con un aditamento esférico de $19.05 \mathrm{~mm}$ de diámetro, el cual se desplazó a una velocidad de $2 \mathrm{~mm} \mathrm{~s}^{-1}$; al entrar en contacto con la tortilla, ésta se estira por la fuerza ejercida hasta que se rompe. El pico máximo de la gráfica representa la fuerza (gf) requerida para romper la tortilla. La distancia $(\mathrm{mm})$ recorrida por la esfera antes de la ruptura de la tortilla corresponde a la elongación (Vázquez-Carrillo et al., 2015).

\section{Análisis estadístico}

Los datos se analizaron conforme a un diseño experimental de bloques completos al azar con arreglo de tratamientos en parcelas subdivididas. Los análisis de varianza, las pruebas de comparación de medias (Tukey, $\mathrm{P} \leq 0.05$ ) y los análisis de correlación simple se hicieron con el programa SAS para Windows, versión 9.0 (SAS Institute, 2002).

\section{RESULTADOS Y DISCUSIÓN}

Se encontraron diferencias significativas $(P \leq 0.01)$ en- tre regímenes hídricos $(\mathrm{H})$ para rendimiento de grano y las propiedades físicas y químicas del grano; entre tipos de madurez $(\mathrm{M})$ para rendimiento de grano $(\mathrm{RG}), \mathrm{PH}$ e IF (P $\leq$ 0.01); entre genotipos $(\mathrm{G})$ para rendimiento de grano, $\mathrm{PH}$, PCG, IF y contenido de proteína $(\mathrm{P} \leq 0.05)$; mientras que la interacción $\mathrm{H} \times \mathrm{M}$ tuvo efecto significativo $(\mathrm{P} \leq 0.05)$ en PH y PCG, y la interacción $\mathrm{H}$ x G solo en $\mathrm{RG}(\mathrm{P} \leq 0.01)$ (Cuadro 2).

En estudios previos realizados también en maíz se ha mostrado que el rendimiento de grano es la característica más afectada por las condiciones de humedad edáfica presentes durante el desarrollo del grano (Pandey et al., 2000; Payero et al., 2006; Kibet et al., 2009; Yi et al., 2010). Los efectos de la interacción $\mathrm{H}$ x G en el rendimiento de grano y de la interacción $\mathrm{H}$ x M en PH y PCG, están relacionados con la duración del periodo de llenado del grano en condiciones favorables de humedad, como lo indicaron Kibet et al. (2009). Para las variables de calidad de nixtamal y tortilla se detectaron diferencias significativas entre regímenes hídricos, en cuanto a porcentaje de sólidos (PS) y elongación (E); entre niveles de madurez hubo diferencias en las variables humedad de nixtamal (HN) y PS; entre genotipos para PS y PR. La interacción $\mathrm{H}$ x M sólo tuvo efecto significativo en la $\mathrm{HN}$, mientras que la interacción $\mathrm{H} \mathrm{x} \mathrm{G}$ solo tuvo efecto significativo en la fuerza de ruptura (FR) (Cuadro 3). 
Cuadro 2. Cuadrados medios y significancias estadísticas para rendimiento y características físicas y químicas del grano de 13 híbridos de maíz con diferente tipo de madurez, cultivados en riego y temporal (secano). Santa Lucía, Estado de México, ciclo Primavera-Verano 2011.

\begin{tabular}{|c|c|c|c|c|c|c|c|c|}
\hline $\mathrm{FV}^{\dagger}$ & $\mathrm{GL}^{\dagger \dagger}$ & $\mathrm{RG}^{\prime}$ & $\mathrm{PH}^{99}$ & $\mathrm{PCG}^{\xi}$ & $\mathrm{IF}^{\xi \xi}$ & Proteína & Almidón & Amilosa \\
\hline Régimen hídrico $(\mathrm{H})$ & 1 & $127.95^{\star *}$ & $14.75^{\star \star}$ & $289.24^{\star *}$ & $2383.78^{\star *}$ & $12.32^{\star *}$ & $185.20^{\star *}$ & $38.53^{* *}$ \\
\hline Madurez (M) & 2 & $29.77^{\star \star}$ & $80.12^{\star *}$ & 57.92 & $2868.34^{\star *}$ & 1.56 & 25.09 & 4.35 \\
\hline Genotipo (G) & 12 & $76.85^{\star *}$ & $13.85^{\star *}$ & $853.84^{\star *}$ & $2576.31^{\star *}$ & $3.22^{*}$ & 16.47 & 7.65 \\
\hline $\mathrm{H} \times \mathrm{G}$ & 12 & $4.69^{* *}$ & 0.99 & 12.29 & 159.47 & 0.35 & 2.70 & 0.97 \\
\hline
\end{tabular}

Cuadro 3. Cuadrados medios y significancias estadísticas para las variables de calidad de nixtamal y tortilla de 13 híbridos de maíz, con diferente tipo de madurez cultivados en riego y temporal (secano). Santa Lucía, Estado de México, ciclo PrimaveraVerano 2011.

\begin{tabular}{|c|c|c|c|c|c|c|c|c|}
\hline $\mathrm{FV}^{\dagger}$ & $\mathrm{GL}^{\dagger \dagger}$ & $\mathrm{HN}^{9}$ & HT & $\mathrm{PS}^{\xi}$ & $\mathrm{PR}^{\xi \xi}$ & $\mathrm{RTF}^{\Phi}$ & $\mathrm{FR}^{\Phi \Phi}$ & Elongación \\
\hline Régimen hídrico $(\mathrm{H})$ & 1 & 1.02 & 4.38 & $0.57^{\star}$ & 10.52 & 0.01 & 359.04 & $5.84^{* *}$ \\
\hline Madurez (M) & 2 & $34.24^{* *}$ & 25.42 & $2.29^{* *}$ & 43.52 & 0.01 & 31.83 & 1.25 \\
\hline $\mathrm{HX} \mathrm{M}$ & 2 & $13.73^{\star}$ & 0.31 & 0.09 & 48.61 & 0.01 & 405.28 & 0.82 \\
\hline Genotipo (G) & 12 & 0.01 & 24.18 & $0.50^{*}$ & $1062.71^{\star *}$ & 0.01 & 2227.26 & 0.23 \\
\hline $\mathrm{H} \times \mathrm{G}$ & 12 & 0.03 & 5.36 & 0.22 & 4.79 & 0.01 & $7242.08^{*}$ & 0.68 \\
\hline
\end{tabular}

${ }^{*} \mathrm{P}<0.05 ;{ }^{* *} \mathrm{P}<0.01 .{ }^{\dagger} \mathrm{FV}=$ fuente de variación; ${ }^{\dagger \dagger} \mathrm{GL}=$ grados de libertad; ${ }^{9} \mathrm{HN}=$ humedad de nixtamal; $9 \mathrm{HT}=$ humedad de tortilla; ${ }^{\xi} \mathrm{PS}=$ peso de sólidos en nejayote; ${ }^{\xi \xi} \mathrm{PR}=$ pericarpio retenido; ${ }^{\Phi} \mathrm{RTF}=$ rendimiento de tortilla fría; ${ }^{\Phi \Phi} \mathrm{FR}$ = fuerza de ruptura.

\section{Régimen hídrico, rendimiento y características físicas y químicas de grano}

En general, todos los híbridos cultivados bajo condiciones de riego presentaron mayor rendimiento de grano que los cultivados bajo condiciones de temporal (Cuadro 4). El menor rendimiento obtenido en temporal se debe principalmente a las deficiencias hídricas que se presentaron durante todo el desarrollo del cultivo, principalmente durante las etapas vegetativa y de llenado de grano (Figura 2). Las necesidades de agua de la planta son mayores durante las primeras etapas del crecimiento, sin embargo, las deficiencias hídricas durante el periodo cercano a la floración y el periodo de llenado de grano pueden reducir en más de 50 $\%$ el rendimiento de grano porque se afecta la fecundación y el desarrollo del grano, lo cual disminuye el número, peso y calidad del grano (NeSmith y Ritchie, 1992; Edreira et al., 2014).

Boyer y Westgate (2004) postularon que en los híbridos sembrados bajo condiciones de temporal la deficiencia de agua inhibe sustancialmente la fotosíntesis que resulta en una escasa acumulación de materia seca. Esto se vio reflejado aquí en el contenido de almidón que fue mayor en los híbridos de riego que en los de temporal (Cuadro 4). Por otro lado, una mayor acumulación de almidón produjo granos con un PH alto, un mayor tamaño de grano y granos más duros. Yang et al. (2000) encontraron que en ambientes con alta humedad el $\mathrm{PH}$ es bajo, y en los de baja precipitación el PH es relativamente alto, pero tal comportamiento no se observó en este experimento.

El contenido de proteína en grano fue afectado por el régimen hídrico y se encontró un mayor contenido de este componente en los híbridos cultivados en temporal respecto a los cultivados en riego (Cuadro 4). De acuerdo con Mohammadkhani y Heidari (2008), el déficit de agua produce cambios en la expresión, acumulación y síntesis de proteínas. En maíz se ha detectado un incremento en la expresión de 50 proteínas, la disminución de 23 y se induce la síntesis de 10 proteínas conocidas como dehidrinas. Estas proteínas se producen en respuesta al déficit de agua durante su desarrollo y juegan un papel importante en la estabilidad de la membrana y la protección de las células contra la deshidratación (Riccardi et al., 1998).

Por su parte el contenido de amilosa siguió la misma tendencia que la dureza del grano, es decir se encontró un mayor contenido de amilosa en los granos de los híbridos cultivados en riego (IF $=61 \%$ ) que en los híbridos cultivados en temporal $(\mathrm{IF}=72 \%)$. Esto coincide con lo reportado por Agama-Acevedo et al. (2013), quienes sugirieron que 
Cuadro 4. Rendimiento, características físicas y químicas de grano de 13 híbridos de maíz en función del régimen hídrico, madurez y genotipo. Santa Lucía, Estado de México, ciclo Primavera-Verano 2011.

\begin{tabular}{|c|c|c|c|c|c|c|c|c|}
\hline $\begin{array}{l}\text { Régimen } \\
\text { hídrico }\end{array}$ & & $\mathrm{RG}^{\dagger}\left(\mathrm{t} \mathrm{ha}^{-1}\right)$ & $\begin{array}{c}\mathrm{PH}^{\dagger \dagger} \\
\left(\mathrm{kg} \mathrm{hL}^{-1}\right)\end{array}$ & $\mathrm{PCG}^{g}(\mathrm{~g})$ & IF99 (\%) & $\begin{array}{c}\text { Proteína } \\
(\%)\end{array}$ & $\begin{array}{c}\text { Almidón } \\
(\%)\end{array}$ & $\begin{array}{c}\text { Amilosa } \\
(\%)\end{array}$ \\
\hline \multicolumn{9}{|c|}{ Análisis por régimen hídrico } \\
\hline Riego & & $8.1 \mathrm{a}$ & $73.8 \mathrm{a}$ & $34.3 \mathrm{a}$ & $61 \mathrm{~b}$ & $10.9 \mathrm{~b}$ & $74.4 \mathrm{a}$ & $27.3 \mathrm{a}$ \\
\hline Temporal & & $5.5 \mathrm{~b}$ & $72.9 \mathrm{~b}$ & $30.4 \mathrm{~b}$ & $72 \mathrm{a}$ & $11.7 \mathrm{a}$ & $71.3 \mathrm{~b}$ & $25.6 \mathrm{~b}$ \\
\hline \multicolumn{9}{|c|}{ Análisis por tipo de madurez } \\
\hline \multirow{4}{*}{ Riego } & Tardío & $7.9 \mathrm{ab}$ & $73.0 \mathrm{~b}$ & $35.8 \mathrm{a}$ & $65 \mathrm{ab}$ & $10.8 \mathrm{~b}$ & $74.1 \mathrm{ab}$ & $27.4 \mathrm{a}$ \\
\hline & Intermedia & $7.5 \mathrm{~b}$ & $74.4 \mathrm{ab}$ & $31.5 \mathrm{a}$ & $55 \mathrm{~b}$ & $10.8 \mathrm{~b}$ & $75.1 \mathrm{a}$ & $27.2 \mathrm{a}$ \\
\hline & Precoz & $9.1 \mathrm{a}$ & $74.2 \mathrm{ab}$ & $35.4 \mathrm{a}$ & $60 \mathrm{~b}$ & $11.2 \mathrm{ab}$ & $73.9 \mathrm{abc}$ & $28.4 \mathrm{a}$ \\
\hline & Tardía & $4.9 \mathrm{~d}$ & $71.3 \mathrm{c}$ & $30.3 \mathrm{a}$ & $80 \mathrm{a}$ & $11.5 \mathrm{ab}$ & $70.9 \mathrm{bc}$ & $26.5 \mathrm{a}$ \\
\hline \multirow[t]{2}{*}{ Temporal } & Intermedia & $5.4 \mathrm{~cd}$ & $74.6 \mathrm{a}$ & $30.6 \mathrm{a}$ & $62 \mathrm{ab}$ & $12.0 \mathrm{a}$ & $72.2 \mathrm{abc}$ & $27.3 \mathrm{a}$ \\
\hline & Precoz & $6.7 \mathrm{bc}$ & $73.4 \mathrm{ab}$ & $30.2 \mathrm{a}$ & $71 \mathrm{ab}$ & $11.7 \mathrm{ab}$ & $70.8 \mathrm{c}$ & $26.9 \mathrm{a}$ \\
\hline \multicolumn{9}{|c|}{ Análisis por genotipos } \\
\hline \multirow{2}{*}{ Riego } & Antiguos & $6.1 \mathrm{~b}$ & $72.5 \mathrm{~b}$ & $29.3 \mathrm{bc}$ & $78 \mathrm{ab}$ & $11.2 \mathrm{a}$ & $74.5 \mathrm{a}$ & $26.6 \mathrm{a}$ \\
\hline & Modernos & $8.7 \mathrm{a}$ & $74.1 \mathrm{a}$ & $35.5 \mathrm{a}$ & $57 c$ & $10.8 \mathrm{a}$ & $74.3 \mathrm{a}$ & $28.1 \mathrm{a}$ \\
\hline \multirow{2}{*}{ Temporal } & Antiguos & $3.9 \mathrm{c}$ & $70.3 \mathrm{c}$ & $25.1 \mathrm{c}$ & $88 \mathrm{a}$ & $11.7 \mathrm{a}$ & $71.9 \mathrm{ab}$ & $25.1 \mathrm{a}$ \\
\hline & Modernos & $6.0 \mathrm{~b}$ & $73.5 \mathrm{ab}$ & $31.6 \mathrm{ab}$ & $68 \mathrm{bc}$ & $11.7 \mathrm{a}$ & $71.1 \mathrm{~b}$ & $26.1 \mathrm{a}$ \\
\hline
\end{tabular}

Medias con letras iguales no son estadísticamente diferentes (Tukey, 0.05). ${ }^{\dagger} \mathrm{RG}=$ rendimiento de grano; ${ }^{\dagger \dagger} \mathrm{PH}=$ peso hectolítrico; ${ }^{\mathrm{P} C G}=$ peso de cien granos; "IF = índice de flotación.

un grano duro presenta un mayor contenido de amilosa que ocasiona que los gránulos de almidón puedan ser comprimidos fácilmente por su matriz proteica, a diferencia de los granos suaves que tienen más amilopectina.

\section{Tipo de madurez, rendimiento y características físicas y químicas de grano}

El análisis por tipo de madurez mostró que, en ambos regímenes hídricos, los híbridos precoces tuvieron mayor rendimiento de grano, $9.1 \mathrm{t} \mathrm{ha}^{-1}$ en riego y $6.7 \mathrm{t} \mathrm{ha}^{-1}$ en temporal, mientras que el rendimiento más bajo se presentó en riego en los híbridos de madurez intermedia y en temporal en los híbridos tardíos. El PH e IF no mostraron diferencias significativas entre los diferentes tipos de madurez en riego y los granos fueron de dureza intermedia ( $I F=38$ a $62 \%)$. En temporal los híbridos de madurez intermedia presentaron el PH más alto $\left(74.6 \mathrm{~kg} \mathrm{hL}^{-1}\right)$ y los granos más duros (IF $=62 \%)$, mientras que los híbridos tardíos fueron menos densos $\left(\mathrm{PH}=71.3 \mathrm{~kg} \mathrm{hL}^{-1}\right)$ y más suaves $(\mathrm{IF}=80 \%)$.

Pok et al. (2009) mencionaron que el peso del grano está determinado por la intensidad y la duración de la acumulación de materia seca durante el llenado del grano. Tal condición pudo haber influido aquí, sobre todo en los de riego debido a que los híbridos precoces tuvieron mejor disponibilidad de humedad durante el periodo de llenado de grano que fue más largo (120 d) que el de los híbridos tardíos (100 d) (Figuras 1 y 2), aunque la acumulación de materia seca también está influenciada por factores ambientales y los niveles de fertilización y nutrición del cultivo (Osaki, 1995; Jacobs y Pearson, 1991).

\section{Genotipos, rendimiento y características físicas y químicas de grano}

Al comparar los híbridos modernos con los híbridos antiguos, tanto en riego como en temporal, se encontró que los híbridos modernos cultivados en riego produjeron el mayor rendimiento de grano $\left(8.7 \mathrm{t} \mathrm{ha}^{-1}\right)$, el PH $\left(74.1 \mathrm{~kg} \mathrm{hL}^{-1}\right)$ y PCG (35.5 g) más altos, así como el IF más bajo (57 \%), lo que indica granos más densos, más grandes y más duros. Por su parte, los híbridos antiguos cultivados en temporal presentaron el rendimiento más bajo $\left(3.9 \mathrm{t} \mathrm{ha}^{-1}\right)$ y los granos menos densos $\left(\mathrm{PH}=70.3 \mathrm{~kg} \mathrm{hL}^{-1}\right)$, más pequeños $(\mathrm{PCG}=25.1 \mathrm{~g})$ y más suaves (IF $=88 \%$ ) (Cuadro 4$)$.

Duvick (1992), Echarte et al. (2004) y Kibet et al. (2009) también reportaron un mayor rendimiento en los híbridos modernos respecto a los híbridos antiguos que evaluaron. Según Echarte et al. (2004), este comportamiento se debe a que los híbridos modernos tienen una mayor estabilidad en el rendimiento de grano y una mayor tolerancia al estrés provocado tanto por alta densidad de plantación como 
por los factores abióticos. Además, el rendimiento alto se asocia con la producción de un mayor número de granos por planta, producto de una mayor asignación de materia seca a la mazorca durante el periodo crítico de crecimiento del grano después de la emergencia de los estigmas. Duvick (1992) también mencionó que los híbridos modernos tienen mayor rendimiento de grano que los híbridos antiguos en condiciones favorables de humedad, situación que se comprobó en este trabajo.

En el presente estudio también se observó que el rendimiento de grano tuvo cierta asociación con la variación en otras características del grano como $\mathrm{PH}(\mathrm{r}=0.38, \mathrm{P}<0.01)$, PCG $(r=0.67, \mathrm{P}<0.01)$ e IF $(\mathrm{r}=-0.38, \mathrm{P}<0.01)$. Similarmente, Vázquez et al. (2012) demostraron que los efectos sobre una variable también son observados en otra. El IF correlacionó con el rendimiento $(\mathrm{r}=-0.38, \mathrm{P}<0.01), \mathrm{PH}$ $(\mathrm{r}=-0.58, \mathrm{P}<0.01)$, y PCG $(\mathrm{r}=-0.25, \mathrm{P}<0.05)$, lo que indica que entre más duros sean los granos, mayor serán el rendimiento, el PH y el tamaño del grano. No obstante, estas correlaciones fueron menores a las informadas por Vázquez et al. (2012).

Desde el punto de vista industrial, tanto la industria de harina para nixtamal (IHN) como la industria de masa para tortilla (IMT) demandan granos con un $\mathrm{PH}$ mayor a $74 \mathrm{~kg} \mathrm{hL}^{-1}$ (SE, 2002), valores que solo se presentaron en los híbridos modernos de madurez intermedia [H-64, H-68, H-70 y H-72] y precoces [HPrecoz-1, HPrecoz-2 y HPrecoz-3] cuando fueron sembrados bajo condiciones de riego. Son aptos para la industria también los híbridos intermedios [H-64, H-68, H-70 y H-72] cultivados en temporal. Sin embargo, ninguno de los materiales evaluados fueron aceptables para la IHN debido a que esta industria requiere granos muy duros y duros (IF $<40 \%$ ). Todos los híbridos presentaron granos de dureza intermedia y suave, por lo que su principal destino es la elaboración de tortillas a partir de masa fresca (IMT).

\section{Régimen hídrico y parámetros de calidad de nixtamal y tortilla}

El régimen hídrico tuvo efectos sobre las variables porcentaje de sólidos y elongación (E), pero sin diferencias significativas por efecto de las interacciones $\mathrm{H}$ x M y $\mathrm{H}$ x G para estas variables (Cuadro 3). El porcentaje de sólidos en el nejayote fue mayor en los híbridos de riego (3.5\%) que en los de temporal (3.3\%) (Cuadro 5), lo que se podría explicar debido a que los granos de los híbridos de riego fueron más duros y requirieron un mayor tiempo de nixtamalización (35 $\mathrm{min}$ ) que los cultivados en temporal (30 $\mathrm{min})$, lo que provocó una mayor hidrólisis del pericarpio $(\mathrm{PR}=$ $40.5 \%$ ) y la lixiviación de mayor cantidad de compuestos del grano como el almidón, amilosa, proteínas y lípidos.

Cuadro 5. Variables de calidad de nixtamal y tortilla de 13 híbridos de maíz en función del régimen hídrico, madurez y genotipo. Santa Lucía, Estado de México, ciclo Primavera-Verano 2011.

\begin{tabular}{|c|c|c|c|c|c|c|c|c|}
\hline \multicolumn{2}{|c|}{ Régimen hídrico } & $\mathrm{HN}^{\dagger}(\%)$ & $\mathrm{HT}^{\dagger \dagger}(\%)$ & PS $^{9}(\%)$ & $\operatorname{PR}^{99}(\%)$ & $\mathrm{RTF}^{\xi}$ & $F R^{\xi \xi}(\mathrm{gf})$ & $\begin{array}{l}\text { Elongación } \\
(\mathrm{mm})\end{array}$ \\
\hline \multicolumn{9}{|c|}{ Análisis por régimen hídrico } \\
\hline \multicolumn{2}{|l|}{ Riego } & $44.0 \mathrm{a}$ & $42.2 \mathrm{a}$ & $3.5 \mathrm{a}$ & $40.5 \mathrm{a}$ & $1.47 \mathrm{a}$ & $192 \mathrm{a}$ & $11.6 \mathrm{a}$ \\
\hline \multicolumn{2}{|l|}{ Temporal } & $44.3 \mathrm{a}$ & $41.7 \mathrm{a}$ & $3.3 \mathrm{~b}$ & $41.3 \mathrm{a}$ & $1.49 \mathrm{a}$ & $196 \mathrm{a}$ & $11.0 \mathrm{~b}$ \\
\hline \multicolumn{9}{|c|}{ Análisis por tipo de madurez } \\
\hline \multirow{3}{*}{ Riego } & Tardía & $44.7 \mathrm{ab}$ & $42.8 \mathrm{a}$ & $3.3 \mathrm{abc}$ & $40.2 \mathrm{a}$ & $1.47 \mathrm{a}$ & $192 \mathrm{a}$ & $11.6 \mathrm{a}$ \\
\hline & Intermedia & $43.8 \mathrm{abc}$ & $41.9 \mathrm{a}$ & $3.7 \mathrm{a}$ & $40.1 \mathrm{a}$ & $1.48 \mathrm{a}$ & $184 \mathrm{a}$ & $11.6 \mathrm{a}$ \\
\hline & Precoz & $43.2 \mathrm{bc}$ & $41.3 \mathrm{a}$ & $3.5 \mathrm{abc}$ & $41.7 \mathrm{a}$ & $1.46 \mathrm{a}$ & $201 \mathrm{a}$ & $11.7 \mathrm{a}$ \\
\hline \multirow{3}{*}{ Temporal } & Tardía & $45.1 \mathrm{a}$ & $42.3 \mathrm{a}$ & $3.1 \mathrm{c}$ & $39.9 \mathrm{a}$ & $1.51 \mathrm{a}$ & $196 \mathrm{a}$ & $11.3 \mathrm{ab}$ \\
\hline & Intermedia & $42.9 \mathrm{c}$ & $41.3 \mathrm{a}$ & $3.6 \mathrm{ab}$ & $43.2 \mathrm{a}$ & $1.47 \mathrm{a}$ & $202 \mathrm{a}$ & $10.8 \mathrm{~b}$ \\
\hline & Precoz & $44.5 \mathrm{abc}$ & $41.1 \mathrm{a}$ & $3.2 \mathrm{bc}$ & $41.2 \mathrm{a}$ & $1.48 \mathrm{a}$ & $188 \mathrm{a}$ & $11.1 \mathrm{ab}$ \\
\hline \multicolumn{9}{|c|}{ Análisis por genotipo } \\
\hline \multirow{2}{*}{ Riego } & Antiguos & $44.7 \mathrm{a}$ & $42.3 \mathrm{a}$ & $3.3 \mathrm{ab}$ & $46.7 \mathrm{a}$ & $1.48 \mathrm{a}$ & $166 \mathrm{a}$ & $11.4 \mathrm{ab}$ \\
\hline & Modernos & $43.9 \mathrm{a}$ & $42.2 \mathrm{a}$ & $3.5 \mathrm{a}$ & $39.1 \mathrm{a}$ & $1.47 \mathrm{a}$ & 198 a & $11.7 \mathrm{a}$ \\
\hline \multirow{2}{*}{ Temporal } & Antiguos & $45.1 \mathrm{a}$ & $40.9 \mathrm{a}$ & $2.9 \mathrm{~b}$ & $46.4 \mathrm{a}$ & $1.51 \mathrm{a}$ & $204 \mathrm{a}$ & $11.4 \mathrm{ab}$ \\
\hline & Modernos & $44.1 \mathrm{a}$ & $41.9 \mathrm{a}$ & $3.4 \mathrm{a}$ & $40.1 \mathrm{a}$ & $1.48 \mathrm{a}$ & $194 \mathrm{a}$ & $11.0 \mathrm{~b}$ \\
\hline
\end{tabular}

Medias con letras iguales no son estadísticamente diferentes (Tukey, 0.05). ${ }^{\dagger} \mathrm{HN}=$ humedad de nixtamal; ${ }^{\dagger \dagger} \mathrm{HT}=$ humedad de tortilla; ${ }^{\mathrm{P}} \mathrm{PS}=$ porcentaje de sólidos en nejayote; " $\mathrm{PR}=$ pericarpio retenido; ${ }^{\xi} \mathrm{RTF}=$ rendimiento de tortilla fría $\left(\mathrm{kg}\right.$ de tortilla/ $\mathrm{kg}$ maíz procesado); ${ }^{\xi \xi} \mathrm{FR}=$ fuerza de ruptura. 
Respecto a la textura de las tortillas, las de híbridos cultivados en riego presentaron mayor elongación $(11.6 \mathrm{~mm})$ y requirieron menor fuerza de ruptura (192 gf), lo que indica que estas tortillas fueron más suaves que las de los híbridos de temporal. La dureza de las tortillas se debe a la retrogradación del almidón, y la amilosa es la principal molécula responsable de este fenómeno, por lo que a mayor contenido de amilosa mayor retrogradación (Agama-Acevedo et al., 2013). Sin embargo, en este estudio los híbridos cultivados en riego, que fueron más duros y tuvieron mayor contenido de amilosa, presentaron las tortillas más suaves, lo que podría deberse a que estos granos requirieron un mayor tiempo de nixtamalización (35 min) y por ello tuvieron una mayor lixiviación de la amilosa que dio lugar a una menor retrogradación y tortillas más suaves.

\section{Tipo de madurez y calidad de nixtamal y tortilla}

La humedad del nixtamal (HN) y el porcentaje de sólidos (PS) en nejayote presentaron diferencias significativas $(\mathrm{P} \leq$ $0.01)$ por efecto del tipo de madurez, y la interacción $\mathrm{H}$ x M afectó significativamente $(\mathrm{P} \leq 0.05)$ a la $\mathrm{HN}$. Esta variable fue mayor en los híbridos tardíos (44.7 a $45.1 \%$ ) que en los precoces e intermedios (42.9 a $44.5 \%$ ) (Cuadro 5), porque los genotipos tardíos presentaron granos más suaves que les permitió una mejor hidratación del endospermo del grano durante el proceso de nixtamalización y el reposo (Salinas et al., 2010). Una alta HN (44.7 a $45.1 \%)$ influyó en una mayor humedad (42.3 a $42.8 \%$ ) y rendimiento de tortillas (1.47 a $1.51 \mathrm{~kg} / \mathrm{kg}$ maíz procesado). No obstante, los rendimientos se consideran bajos ya que la industria de la masa para tortillas (IMT) espera obtener rendimientos mayores a $1.5 \mathrm{~kg}$ de tortilla por kg de maíz.

En cuanto al porcentaje de sólidos en nejayote, que refleja la cantidad de materia seca perdida por efecto del proceso de nixtamalización, se observó que los granos de los híbridos tardíos (suaves; IF $=65$ a $80 \%$ ) tuvieron una menor pérdida de materia seca ( $P S=3.1$ a $3.3 \%$ ), atribuible a que tuvieron un menor tiempo de cocimiento $(30 \mathrm{~min})$ y por tanto menor lixiviación de compuestos durante el proceso de nixtamalización. En cambio, los híbridos precoces e intermedios menos suaves ( $\mathrm{IF}=55$ a $71 \%$ ) y que requirieron 35 min de cocción, perdieron más sólidos ( $\mathrm{PS}=3.2$ a $3.7 \%$ ).

\section{Genotipos y calidad de nixtamal y tortilla}

El porcentaje de pericarpio retenido en el nixtamal (PR) y el porcentaje de sólidos (PS) en nejayote fueron las variables más afectadas por el genotipo. Los híbridos antiguos retuvieron más pericarpio (46.4 a $46.7 \%$ ) que los híbridos modernos (41.9 a $42.3 \%$ ), lo que provocó un menor PS en los híbridos antiguos (2.9 a $3.3 \%$ ) respecto a los modernos (3.4 a 3.5 \%) (Cuadro 5); en ambos casos el PS se encontró dentro del límite máximo de $5 \%$ permitido por la industria (Vázquez et al., 2012).

De acuerdo con Serna et al. (2008), la retención de pericarpio al grano es una característica heredable y usualmente los granos más pequeños retienen más pericarpio que los grandes. Aquí se encontró que los híbridos antiguos presentaron en promedio más pericarpio (5.9\%) que los modernos (5.6\%), lo cual favorece este comportamiento. Una mayor retención de pericarpio en nixtamal mejora la textura de la masa y de las tortillas, debido a la presencia de arabinoxilanos y demás gomas naturales que se liberan por la hidrólisis del pericarpio durante el proceso de nixtamalización. Este efecto se observó en los híbridos cultivados bajo riego en donde las tortillas de los híbridos antiguos fueron numéricamente más suaves al tener menor fuerza de ruprura ( $F R=166$ gf $)$ que las de los híbridos modernos (FR = 198 gf) (Cuadro 5).

\section{CONCLUSIONES}

La interacción entre el genotipo y el régimen hídrico influyó en el rendimiento de grano y las características físicas y químicas del mismo. Las deficiencias hídricas que se presentaron durante todo el periodo de cultivo en los híbridos cultivados en temporal provocaron bajos rendimientos (5.5 $\mathrm{t} \mathrm{ha}^{-1}$ ), efecto que se asoció con granos suaves, pequeños, y con bajo peso hectolítrico, con respecto a los cultivados en riego $\left(8.1 \mathrm{t} \mathrm{ha}^{-1}\right)$. Los híbridos modernos tienen mayor rendimiento de grano, así como ganancias en el peso hectolítrico, el tamaño de los granos y su dureza, en comparación con los híbridos antiguos; sin embargo, el avance en mejoramiento genético se reflejó poco en la calidad de las tortillas. Todos los híbridos evaluados son aptos para su procesamiento por la industria de la masa y la tortilla y para autoconsumo. Los híbridos antiguos cultivados bajo temporal registraron la menor pérdida de sólidos en el nejayote $(2.9 \%)$ y numéricamente el mayor rendimiento de tortillas (1.51 kg de tortilla/kg de maíz).

\section{BIBLIOGRAFÍA}

AACC, American Association of Cereal Chemists (2000) Approved Methods of the AACC. 16th ed. AACC. The Association. St. Paul, MN. USA. 1200 p.

Agama-Acevedo E., E. Juárez-García, S. Evangelista-Lozano, O. L. Rosales-Reynoso y L. A. Bello-Pérez (2013) Características del almidón de maíz y relación con las enzimas de su biosíntesis. Agrociencia 47:1-12.

Arellano V. J. L., F. Castillo G., G. Alcántara G. y A. Martínez G. (1996) Parámetros genéticos de la eficiencia en el uso de nitrógeno en líneas de maíz de Valles Altos. In: Developing Drought- and Low N-Tolerant Maize. G. O. Edmeades, M. Banziger, H. T. Mickelson and C. B. Peña-Valdivia (eds). CIMMYT. El Batán, Texcoco, México. pp:320-325.

Boyer J. S. and M. E. Westgate (2004) Grain yields with limited water. Journal of Experimental Botany 55:2385-2394.

Duvick D. N. (1992) Genetic contributions to advances in yield of U.S. 
maize. Maydica 37:69-79.

Echarte L., F. H. Andrade, C. R. C. Vega and M. Tollenaar (2004) Kernel number determination in Argentinean maize hybrids released between 1965 and 1993. Crop Science 44:1654-1661.

Edreira J. I. R., L. I. Mayer and M. E. Otegui (2014) Heat stress in temperate and tropical maize hybrids: Kernel growth, water relations and assimilate availability for grain filling. Field Crops Research 166:162-172.

Galicia L., A. Miranda, M. G. Gutiérrez, O. Custodio, A. Rosales, N. Ruiz, R. Surles y N. Palacios (2012) Laboratorio de Calidad Nutricional de Maíz y Análisis de Tejido Vegetal. Protocolos de Laboratorio. Centro Internacional de Mejoramiento de Maíz y Trigo. México, D.F. 50 p.

Hoover R. and W. S. Ratnayake (2001) Determination of total amylose content of starch. Current Protocols of Food Analytical Chemistry E2.3:1-5.

Jacobs B. C. and C. J. Pearson (1991) Potential yield of maize, determined by rates of growth and development of ears. Field Crops Research 27:281-298.

Kibet S. C., C. López C. y J. Kohashi S. (2009) Efecto del nivel de humedad y nitrógeno en el suelo en el comportamiento de maíces híbridos y criollos de los Valles Altos de México. Agronomía Costarricense 33:103-120.

Mohammadkhani N. and R. Heidari (2008) Effects of drought stress on soluble proteins in two maize varieties. Turkish Journal of Bio$\log$ 32:23-30.

NeSmith D. S. and J. T. Ritchie (1992) Maize (Zea mays L.) response to a severe soil water-deficit during grain filling. Field Crops Research 29:23-55.

Osaki M. (1995) Comparison of productivity between tropical and temperate maize. II. Parameters determining the productivity in relation to the amount of nitrogen absorbed. Soil Science and Plant Nutrition 41:451-459.

Pandey R. K., J. W. Maranville and A. Admou (2000) Deficit irrigation and nitrogen effects on maize in a Sahelian environment I. Grain yield and yield components. Agricultural Water Management 46:1-13.

Payero J. O., S. R. Melvin, S. Irmak and D. Tarkalson (2006) Yield response of corn to deficit irrigation in a semiarid climate. Agricultural Water Management 84:101-112.

Pok I., T. Spitko and J. Pinter (2009) Effects of environmental factors on the grain filling of maize genotypes. Maydica 54:259-262.

Riccardi F., P. Gazeau, D. Vienne and M. Zivy (1998) Protein changes in response to progressive water deficit in maize. Plant Physiology 117:1253-1263.

Salinas M. Y., N. O. Gómez M., J. E. Cervantes M., M. Sierra M., A. Palafox C., E. Betanzos M. and B. Coutiño E. (2010) Calidad nixtamalera y tortillera en maíces del trópico húmedo y subhúmedo de México. Revista Mexicana de Ciencias Agrícolas 1:509-523.

SAS, Statistical Analysis System (2002) The SAS System for Windows 9.0 . User's guide. Cary, N. C. USA. 584 p.

SE, Secretaría de Economía (2002) NMX-FF-034/1-SCFI-2002. Productos alimenticios no industrializados para consumo humano -Cereales-Parte I: Maíz blanco para proceso alcalino para tortillas de maíz y productos de maíz nixtamalizado-Especificaciones y métodos de prueba. Dirección General de Normas. México, D. F. 18 p.

Serna S. S. O., C. A. Amaya G., P. Herrera M., J. L. Melesio C., R. E. Preciado O., A. D. Terrón I. nad G. Vázquez C. (2008) Evaluation of the limecooking and tortilla making properties of quality protein maize hybrids grown in Mexico. Plant Foods for Human Nutrition 63:119-125.

SIAP, Servicio de Información Agroalimentaria y Pesquera (2012) Cierre de la Producción Agrícola por Cultivo. SIAP-SAGARPA. Disponible en: http://www.siap.gob.mx/ (Diciembre 2013).

Vázquez-Carrillo G., S. Garcia-Lara, Y. Salinas-Moreno, D. J. Bergvinson and N. Palacios-Rojas (2011) Grain and tortilla quality in landraces and improved maize grown in the highlands of Mexico. Plant Foods for Human Nutrition 66:203-208

Vázquez C. M. G., D. Santiago R., Y. Salinas M., I. Rojas M., J. L. Arellano V., G. A. Velázquez C. and A. Espinoza C. (2012) Interacción genotipo-ambiente del rendimiento y calidad de grano y tortilla de híbridos de maíz en Valles Altos de Tlaxcala, México. Revista Fitotecnia Mexicana 35:229-237.

Vázquez-Carrillo M. G., D. Santiago-Ramos, M. Gaytán-Martínez, E. Morales-Sánchez and M. J. Guerrero-Herrera (2015) High oil content maize: Physical, thermal and rheological properties of grain, masa, and tortillas. LWT-Food Science and Technology 60:156-1613.

Yang P., R. J. Shunk, A. E. Haken, Y. X. Niu, S. H. Zou, P. Buriak, S. R. Eckhoff and E. Tumbleson (2000) Yield, protein content, and viscosity of starch from wet-milled corn hybrids as influenced by environmentally induced changes in test weight. Cereal Chemistry 77:44-47.

Yi L., Y. Shenjiao, L. Shiqing, C. Xinping and C. Fang (2010) Growth and development of maize (Zea mays L.) in response to different field water management practices: Resource capture and use efficiency. Agricultural and Forest Meteorology 150:606-613.

Zepeda B. R., A. Carballo C., A. Muñoz O., J. A. Mejía C., B. Figueroa S. y F. V. González C. (2007) Fertilización nitrogenada y características físicas, estructurales y calidad de nixtamal-tortilla del grano de híbridos de maíz. Agricultura Técnica de México 33:17-24. 